\title{
Efeito de grãos conilon no perfil sensorial e aceitação de bebidas de café
}

\section{Effect of conilon beans on the sensory profile and consumer acceptance of coffee beverages}

\author{
Eliana de Souza Marques dos Santos ${ }^{1,2 *}$; Rosires Deliza ${ }^{3}$; \\ Daniela De Grandi Castro Freitas ${ }^{3}$; Fábio Mathias Corrêa ${ }^{4}$
}

\section{Resumo}

O presente estudo teve como objetivo avaliar as características sensoriais e a aceitabilidade de bebidas de café preparadas a partir de diferentes blends de grãos cafés arábica e conilon. Foram avaliadas bebidas contendo as proporções de zero (100\% arábica), 10, 20, 40, 60, 80 e $100 \%$ de conilon. O perfil sensorial das bebidas foi descrito por uma equipe composta de oito avaliadores selecionados e treinados através do método de Análise Descritiva Quantitativa (ADQ). A aceitabilidade foi avaliada por 112 consumidores de café utilizando-se a escala hedônica de nove pontos. Os dados do teste de aceitabilidade foram submetidos à Análise de Cluster e Mapa de Preferência. Os resultados mostraram que houve uma satisfatória aceitação dos consumidores para as bebidas na proporção de até $40 \%$ de grãos conilon. O estudo mostrou, no entanto, que as bebidas com até $20 \%$ de grãos conilon mantiveram características sensoriais tradicionalmente mais positivas na bebida, como atributos mais intensos de aroma chocolate, aroma e sabor característico de café, aroma e gosto doce.

Palavras-chave: C. arabica, C. canephora, blends, análise descritiva quantitativa, preferência

\begin{abstract}
The present study aimed to evaluate the sensory characteristics and acceptability of coffee beverages prepared from different blends of arabica beans and conilon coffee beans. Beverages with different proportions of conilon, containing zero (100\% arabica), 10, 20, 40, 60, 80 and $100 \%$ of conilon were evaluated. The sensory profile of the coffee brews was described by eight selected and trained assessors using the Quantitative Descriptive Analysis (QDA). The beverages acceptance was evaluated by 112 coffee consumers using nine-point hedonic scales. The results were submitted to the Cluster Analysis and Preference Mapping. A satisfactory acceptance for the beverages up to $40 \%$ of conilon beans was observed. The study showed, however, that beverages contained up to $20 \%$ of conilon beans kept the sensory characteristics traditionally considered as more positive for coffee brews, such as high intensity of chocolate aroma, coffee aroma and flavor, sweet aroma, and sweet taste.
\end{abstract}

Key words: C. arabica, C. canephora, blends, quantitative descriptive analysis, preference

\footnotetext{
${ }^{1}$ Prof ${ }^{a}$ do Instituto Federal de Educação, Ciência e Tecnologia do Rio de Janeiro, IFRJ, Pinheiral, RJ. E-mail: eliana.santos@ifrj. edu.br

${ }^{2}$ Discente, Dept ${ }^{\circ}$ de Tecnologia de Alimentos, Universidade Federal Rural do Rio de Janeiro, UFRRJ, Seropédica, RJ.

${ }^{3}$ Pesquisadoras, Empresa Brasileira de Pesquisa Agropecuária, EMBRAPA, Centro Nacional de Pesquisa de Tecnologia Agroindustrial de Alimentos, CTAA, Rio de Janeiro, RJ. E-mail: rosires.deliza@embrapa.br; daniela@ctaa.embrapa.br

${ }^{4}$ Prof. do Dept ${ }^{\circ}$ de Ciências Exatas e da Terra da Universidade Estadual de Santa Cruz, UESC, Ilhéus, BA. E-mail: fncrom@gmail. com

* Autor para correspondência
} 


\section{Introdução}

As duas principais espécies de café, Coffea arabica e Coffea canephora, diferem consideravelmente quanto ao preço, qualidade e aceitação pelo consumidor. As bebidas proporcionadas por cada uma dessas espécies são diferentes, assim como a preferência do grande mercado consumidor. Blends com estas duas espécies tem se tornado uma opção interessante para se obter um bom produto, que agrade a maioria dos consumidores e aproveite o máximo das caracteristicas de qualidade de ambas as espécies. O café arábica tem sido usado nos blends pelo efeito benéfico em relação ao aroma e sabor, enquanto o conilon é utilizado pelo incremento do corpo e rentabilidade (MENDES; MENEZES; DA SILVA, 2001; SANZ et al., 2002). Desta forma, as características sensoriais proporcionadas pelo café arábica aliada às características de corpo e rentabilidade advindas do café conilon proporcionam incremento sensorial e redução de custos na produção final do produto industrializado. No entanto, é preciso compreender até que ponto a mistura destas duas espécies são decisivas na preferência do heterogêneo mercado consumidor. Arruda, Ferreira e Minim (2007) destacaram que consumidores estão cada vez mais educados, exigentes e dispostos a pagar mais por um bom café, privilegiando a marca e a qualidade da bebida consumida.

Vários estudos sobre as características sensoriais de café já foram realizados (MOURA et al., 2007; BHUMIRATANA; ADHIKARI; CAHAMBERS IV, 2011; CHALFOUN et al., 2013; SCHOLZ et al., 2013). Na maioria deles foi feita a avaliação da bebida de café de forma isolada e testes afetivos foram discutidos com menor ênfase na aceitação de blends. Tais testes são normalmente realizados após os descritivos e avaliam o grau de gostar e desgostar de um produto, ou ainda a preferência do consumidor por um produto com relação a outro (STONE; SIDEL, 2004), sendo útil na identificação de novos mercados e na modificação dos produtos já existentes.

Com intuito de avaliar os dados afetivos considerando a resposta individual de cada consumidor e não somente a média do grupo que testou os produtos foi desenvolvida a técnica denominada Mapa de Preferência (MacFIE, 1990; SCHLICH; McEWAN, 1992; WESTAD; HERSLETH; LEA, 2004) a qual tem como objetivo identificar grupos de consumidores que respondam semelhantemente facilitando a interpretação dos resultados de acordo com a idade, sexo, atitude, necessidade, hábitos alimentares (WESTAD; HERSLETH; LEA, 2004).

Tendo em vista a crescente busca de consumidores por melhores bebidas de café e a expansão do setor, torna-se oportuno estudar as características sensoriais das referidas bebidas. O objetivo deste estudo foi avaliar sensorialmente blends preparados com grãos arábica e conilon visando identificar bebidas mais apreciadas pelo consumidor e suas respectivas características sensoriais.

\section{Material e Métodos}

Utilizou-se cafés de diferentes espécies. Café arábica (bebida mole) do Tipo 2, com 11,9\% de umidade, cor verde claro e peneiras $15 / 64$, e café conilon (bebida regular) Tipo 6, com 12,6\% de umidade, cor marrom e peneiras $13 / 64$, ambos da safra 2008/2009, das regiões de Franca/SP e Colatina/ ES, respectivamente. Os grãos de cada espécie foram torrados separadamente em torrador elétrico industrial Leogap ${ }^{\circledR}$, modelo Copacabana T-10 por 12 min. $/ 210^{\circ} \mathrm{C}$ (arábica) e por $13 \mathrm{~min} . / 215^{\circ} \mathrm{C}$ (conilon), o que permitiu que ambos atingissem a tonalidade de torração média clara quando comparados com o padrão dos discos colorimétricos do Sistema AGTRON/SCAA Roast Color Classification System (STAUB, 1995). Posteriormente à torração, os grãos foram moídos em moinho (Mecamau ${ }^{\circledR}$ São José Ltda, modelo T368) na granulometria média (predominância de partículas de $0,84 \mathrm{~mm}$ ), para então realizar a formulação dos blends.

Os sete blends avaliados continham zero (100\% arábica), 10, 20, 40, 60, 80 e 100\% de conilon (representadas pelas siglas A100, C10, C20, C40, C60, C80 e C100, respectivamente). O preparo 
das bebidas foi realizado na proporção 1:10 (café : água mineral) (PANGBORN, 1982) em cafeteiras elétricas (marca Britânia ${ }^{\circledR}$, tipo $\mathrm{NCB} 27$ e filtro de papel $\left.\mathrm{n}^{\circ} 103\right)$. As bebidas foram servidas à $70^{\circ} \mathrm{C} \pm$ $2{ }^{\circ} \mathrm{C}$. Apenas no teste de aceitação foi permitido o uso de açúcar refinado, adoçante ou nada, conforme o consumo habitual do participante. Para a condução da análise sensorial, o projeto foi submetido e aprovado pelo Comitê de Ética em Pesquisa envolvendo Seres Humanos da Universidade Veiga de Almeida (Resolução $n^{\circ}$. 132/08).

A pré-seleção de avaliadores foi feita com 12 indivíduos de ambos os sexos e idade entre 20 a 55 anos. $\mathrm{O}$ poder discriminativo para o gosto amargo de cada candidato foi avaliado utilizando a análise sequencial e testes triangulares (MEILGAARD; CIVILLE; CARR, 1999) com probabilidade de erro $\left(\mathrm{p}_{0}\right)=35 \%$, probabilidade de acertos $\left(\mathrm{p}_{1}\right)=65 \%, \alpha=$
$1 \%, \beta=5 \%$. O levantamento dos atributos sensoriais das bebidas de café foi realizado durante dois dias em sessões abertas as quais duraram cerca de $1 \mathrm{~h}$, de acordo com o descrito por Stone et al. (1974). Doze avaliadores provaram todas as bebidas, registraram os atributos sensoriais percebidos em cada amostra e elaboraram uma lista dos atributos e suas respectivas referências identificadas a partir de um conjunto de amostras (Tabela 1). O treinamento foi realizado no período de um mês, avaliando dois atributos por sessão. Verificou-se a discriminação entre as amostras e a repetibilidade dos avaliadores através da avaliação de três amostras distintas com três repetições por indivíduo. $\mathrm{O}$ delineamento experimental utilizado foi o de blocos ao acaso, sendo cada julgador considerado um bloco (8 avaliadores x 3 amostras x 3 repetições). Foi realizada a análise de variância no software R. Development Core Team (Version 2.10.1, Vienna, 2009).

Tabela 1. Atributos sensoriais levantados pela equipe de avaliadores, suas respectivas definições e referências que ancoraram os extremos da escala não estruturada utilizada durante a fase de treinamento na ADQ de sete amostras de bebida de café.

\begin{tabular}{|c|c|c|}
\hline Atributo & Definição & Referência das bebidas \\
\hline $\begin{array}{l}\text { Aroma de } \\
\text { Chocolate }\end{array}$ & Associado ao aroma de chocolate & $\begin{array}{l}\text { Ausente: Bebida } 100 \% \text { conilon. } \\
\text { Forte: Bebida } 100 \% \text { arábica (mole) }\end{array}$ \\
\hline $\begin{array}{l}\text { Aroma e Sabor } \\
\text { Característico }\end{array}$ & $\begin{array}{l}\text { Aroma e sabor de café. Ausência } \\
\text { de aromas e sabores estranhos }\end{array}$ & $\begin{array}{l}\text { Fraco: Bebida } 100 \% \text { conilon. } \\
\text { Forte: Bebida } 100 \% \text { arábica (mole) }\end{array}$ \\
\hline $\begin{array}{l}\text { Aroma e Gosto } \\
\text { Doce }\end{array}$ & $\begin{array}{l}\text { Percepção da doçura associada } \\
\text { aos açúcares presentes }\end{array}$ & $\begin{array}{l}\text { Fraco: Bebida } 100 \% \text { conilon. } \\
\text { Forte: Bebida } 100 \% \text { arábica (mole) }\end{array}$ \\
\hline $\begin{array}{l}\text { Aroma e Sabor } \\
\text { Velho }\end{array}$ & $\begin{array}{l}\text { Odor e sabor de grãos antigos } \\
\text { armazenados em saco de estopa }\end{array}$ & $\begin{array}{l}\text { Ausente: Bebida } 100 \% \text { arábica (mole) } \\
\text { Forte: Bebida } 100 \% \text { conilon safra } 2005\end{array}$ \\
\hline $\begin{array}{l}\text { Aroma e Sabor } \\
\text { Cereal }\end{array}$ & $\begin{array}{l}\text { Aroma semelhante ao milho ou } \\
\text { palha. }\end{array}$ & $\begin{array}{l}\text { Ausente: Bebida } 100 \% \text { arábica (mole) } \\
\text { Forte: Bebida com } 100 \% \text { conilon }\end{array}$ \\
\hline Gosto Ácido & $\begin{array}{l}\text { Associado a percepção de acidez } \\
\text { refrescante e efervescente na } \\
\text { bebida }\end{array}$ & $\begin{array}{l}\text { Fraco: Bebida } 100 \% \text { conilon } \\
\text { Forte: Bebida } 100 \% \text { arábica (mole) adicionado de } 0,05 \% \\
\text { de ácido cítrico monohidratado PA (ACS do Grupo química) }\end{array}$ \\
\hline Gosto Amargo & Associado ao amargor da bebida & $\begin{array}{l}\text { Fraco: Bebida } 100 \% \text { arábica (mole) } \\
\text { Forte: Bebida } 100 \% \text { conilon com adição de } 0,1 \% \text { de cafeína } \\
\text { (Merck) }\end{array}$ \\
\hline $\begin{array}{l}\text { Sensação na boca } \\
\text { de Adstringência }\end{array}$ & $\begin{array}{l}\text { Associado à sensação de secura } \\
\text { deixada na boca após a ingestão }\end{array}$ & $\begin{array}{l}\text { Ausente: Bebida } 100 \% \text { arábica mole. } \\
\text { Muito: Bebida } 100 \% \text { arábica (dura) adicionado de } 0,1 \% \text { de } \\
\text { ácido tânico (Merck) }\end{array}$ \\
\hline $\begin{array}{l}\text { Sensação na boca } \\
\text { de Corpo }\end{array}$ & $\begin{array}{l}\text { Sensação tátil de viscosidade, } \\
\text { preenchimento percebido na boca }\end{array}$ & $\begin{array}{l}\text { Pouco: Água morna } \\
\text { Muito: Bebida } 100 \% \text { conilon }\end{array}$ \\
\hline
\end{tabular}

Fonte: Santos (2010). 
Após o treinamento foi avaliado o desempenho da equipe. Oito avaliadores que apresentam poder discriminativo ( $\mathrm{p} \mathrm{F}_{\text {amostras }}<0,30$ ); reprodutibilidade nos julgamentos ( $\mathrm{p} \mathrm{F}_{\text {repeticōes }} \geq 0,05$ ) e consenso com o grupo (DAMÁSIO; COSTELL, 1991) integraram a equipe definitiva, os quais posteriormente avaliaram as sete bebidas de café seguindo delineamento de blocos incompletos (COCHRAN; COX, 1957), através da ADQ. As bebidas foram servidas monadicamente em xícaras de porcelana branca codificadas com números de três dígitos e a intensidade de cada atributo foi avaliada em escala não estruturada de $9 \mathrm{~cm}(0$ : ausente; 1 : fraco, pouco; 9: forte, muito). Os dados foram coletados através do software Fizz (BIOSYSTEM, 2005), submetidos à análise de variância e as médias checadas pelo teste de Fisher (LSD) $(\alpha=0,05 \%)$, o qual, por ser menos conservador, permite identificar pequenas diferenças entre as amostras. As médias dos atributos sensoriais calculadas com os dados das replicatas e dos avaliadores foram inseridas na matriz de covariância da análise de componentes principais (ACP) usando o software XLSTAT versão 9.0 (ADDINSOFT, 2009).

A aceitação das bebidas foi avaliada por 112 consumidores de café (HOUGH et al., 2006) de ambos os sexos, com idade entre 18 e 55 anos. Utilizou-se a escala hedônica estruturada de nove pontos e todas as bebidas foram avaliadas em uma sessão seguindo o delineamento em blocos completos balanceados (MacFIE et al., 1989). Os dados coletados pelo software Fizz (BIOSYSTEM, 2005) foram analisados por ANOVA e teste de médias de Fisher (LSD) ( $\alpha$ $=0,05 \%)$, por análise de agrupamento e Mapa da Preferência (GREENHOFF; MacFIE, 1994).

\section{Resultados e Discussão}

A equipe de avaliadores decidiu por consenso avaliar o aroma e o sabor conjuntamente utilizando um único atributo. Os atributos avaliados foram: aroma e sabor característico, aroma e sabor velho, aroma e sabor cereal, aroma e gosto doce, aroma de chocolate, gosto ácido, gosto amargo, adstringência e corpo. Houve diferenças entre as bebidas em relação aos atributos avaliados cujas médias são mostradas na Tabela 2 .

A bebida A100 distinguiu-se das demais por ser mais intensa no aroma de chocolate $(8,7)$, aroma e sabor característico $(8,5)$ e aroma e gosto doce $(7,9)$ e ácido $(5,2)$, menos intensa no aroma e sabor velho $(0,4)$ e cereal $(0,5)$ e menos adstringente $(1,1)$. A bebida $\mathrm{C} 100$ apresentou maior intensidade para aroma e sabor velho $(6,6)$ e cereal $(6,7)$, gosto amargo $(5,3)$ e corpo $(5,2)$. Vale ressaltar que o atributo sabor velho diferiu apenas entre a bebida C100 (100\% conilon) e as demais. Tal resultado pode ser devido à variabilidade das notas atribuídas pelos avaliadores para tal atributo sensoriaol. O blend $\mathrm{C} 10$ distinguiu-se do $\mathrm{C} 20$ apenas no aroma e sabor característico (5,6 e 6,8, respectivamente) e gosto amargo (4,5 e 3,6, respectivamente). As amostras C40, C60 e C80 apresentaram perfis semelhantes, como baixa intensidade de aroma chocolate (2,2, 1,3 e 1,2, respectivamente), aroma e sabor característico $(3,8,2,6$ e 2,5, respectivamente), aroma e gosto doce $(3,8,2,5$ e 2, 1 , respectivamente), e maior intensidade de aroma e sabor cereal $(4,2,5,8$ e 6,5 , respectivamente) e adstringência $(3,1,3,2$ e 3,5 , respectivamente) do que as bebidas com menor quantidade de conilon. Entretanto, as bebidas C60 e C80 alcançaram intensidades menores no aroma e sabor característico (2,6 e 2,5, respectivamente) e gosto ácido (2,7 e 2,8, respectivamente); além de maior intensidade no aroma e sabor cereal $(5,8$ e 6.5 , respectivamente), não diferindo $(p>0,05)$ da bebida C100 (6,7) (Tabela 2). Segundo Moura et al. (2007) o aumento da quantidade de grãos conilon no blend ocasionou redução da doçura e acidez e aumento do amargor nas bebidas. O gosto amargo percebido com o aumento da quantidade de grãos do café conilon pode ser resultante da contribuição da cafeína e da degradação térmica dos ácidos clorogênicos, presente em maior concentração nos grãos conilon, que resultam em compostos fenólicos elevando o gosto amargo (CLIFFORD, 1985). 
Tabela 2. Média dos atributos ${ }^{\S}$ sensoriais das bebidas de café obtidas na ADQ.

\begin{tabular}{|c|c|c|c|c|c|c|c|}
\hline \multirow[b]{2}{*}{ Atributos } & \multicolumn{7}{|c|}{ Bebidas } \\
\hline & $\begin{array}{c}100 \% \\
\text { arábica } \\
(\mathrm{A} 100)\end{array}$ & $\begin{array}{c}10 \% \\
\text { conilon } \\
(\mathrm{C} 10)\end{array}$ & $\begin{array}{c}20 \% \\
\text { conilon } \\
\text { (C20) }\end{array}$ & $\begin{array}{c}40 \% \\
\text { conilon } \\
(\mathrm{C} 40)\end{array}$ & $\begin{array}{c}60 \% \\
\text { conilon } \\
(\mathrm{C60})\end{array}$ & $\begin{array}{c}80 \% \\
\text { conilon } \\
(\mathbf{C 8 0})\end{array}$ & $\begin{array}{l}100 \% \\
\text { conilon } \\
(\mathrm{C} 100)\end{array}$ \\
\hline $\begin{array}{l}\text { Aroma de } \\
\text { chocolate }\end{array}$ & $8,7^{\mathrm{a}}$ & $5,5^{\mathrm{b}}$ & $4,7^{\mathrm{b}}$ & $2,2^{\mathrm{c}}$ & $1,3^{\mathrm{c}}$ & $1,2^{\mathrm{c}}$ & $1,7^{\mathrm{c}}$ \\
\hline $\begin{array}{l}\text { Aroma/Sabor } \\
\text { característico }\end{array}$ & $8,5^{\mathrm{a}}$ & $5,6^{\mathrm{c}}$ & $6,8^{\mathrm{b}}$ & $3,8^{\mathrm{d}}$ & $2,6^{\mathrm{e}}$ & $2,5^{\mathrm{e}}$ & $2,5^{\mathrm{e}}$ \\
\hline $\begin{array}{l}\text { Aroma/Gosto } \\
\text { doce }\end{array}$ & $7,9^{\mathrm{a}}$ & $5,4^{\mathrm{b}}$ & $6,3^{b}$ & $3,8^{c}$ & $2,5^{\mathrm{d}}$ & $2,1^{\mathrm{d}}$ & $2,0^{\mathrm{d}}$ \\
\hline $\begin{array}{l}\text { Aroma/Sabor } \\
\text { velho }\end{array}$ & $0,4^{\mathrm{b}}$ & $1,5^{\mathrm{b}}$ & $0,4^{\mathrm{b}}$ & $1,0^{\mathrm{b}}$ & $0,9^{\mathrm{b}}$ & $1,4^{b}$ & $6,6^{\mathrm{a}}$ \\
\hline $\begin{array}{l}\text { Aroma/Sabor } \\
\text { de cereal }\end{array}$ & $0,5^{\mathrm{d}}$ & $2,7^{\mathrm{c}}$ & $2,3^{c}$ & $4,2^{\mathrm{b}}$ & $5,8^{\mathrm{a}}$ & $6,5^{\mathrm{a}}$ & $6,7^{\mathrm{a}}$ \\
\hline Gosto ácido & $5,2^{\mathrm{a}}$ & $4,8^{\mathrm{ab}}$ & $4,2^{\mathrm{b}}$ & $3,4^{c}$ & $2,7^{\mathrm{d}}$ & $2,8^{\mathrm{d}}$ & $3,1^{\mathrm{cd}}$ \\
\hline Gosto amargo & $4,5^{\mathrm{b}}$ & $4,5^{\mathrm{b}}$ & $3,6^{\mathrm{c}}$ & $4,1^{\mathrm{b}}$ & $4,2^{\mathrm{b}}$ & $3,5^{\mathrm{c}}$ & $5,3^{\mathrm{a}}$ \\
\hline Adstringência & $1,1^{\mathrm{d}}$ & $1,8^{\mathrm{c}}$ & $2,1^{\mathrm{c}}$ & $3,1^{b}$ & $3,2^{\mathrm{ab}}$ & $3,5^{\mathrm{a}}$ & $2,1^{\mathrm{c}}$ \\
\hline Corpo & $4,5^{\mathrm{cd}}$ & $4,5^{\mathrm{cd}}$ & $4,3^{\mathrm{d}}$ & $4,8^{\mathrm{bc}}$ & $4,7^{\mathrm{bc}}$ & $5,0^{\mathrm{ab}}$ & $5,2^{\mathrm{a}}$ \\
\hline
\end{tabular}

Médias na mesma linha seguidas de letras diferentes indicam diferença significativa $(\mathrm{p}<0,05)$ pelo teste de Fisher. ${ }^{\S}$ avaliados em escala não estruturada de nove pontos.

Fonte: Santos (2010).

O gosto ácido nos blends (Tabela 2) decresceu à medida que aumentou a quantidade de grãos conilon, havendo redução $(\mathrm{p}<0,05)$ a partir do blend C20. Moura et al. (2007) encontraram diferenças no gosto ácido a partir da adição de $30 \%$ conilon. Pereira et al. (2000) encontraram resultados similares evidenciando que tal aumento foi decorrente da acidez e do teor de compostos fenólicos, os quais tendem ficar mais intensos com o incremento do café conilon na bebida.

Observou-se nos blends C40, C60 e C80 maiores intensidades de adstringência (3,1, 3,2 e 3,5, respectivamente); no entanto, não identificadas como um aumento gradual quando comparado à bebida C100 (2,1) (Tabela 2). Deve-se considerar, no entanto, que De Maria et al. (1995) observaram que os ácidos clorogênicos presentes em maior concentração nos grãos conilon contribuem para a adstringência.

A ACP resultou numa solução bidimensional e explicou $96 \%$ da variação dos dados (Figura 1).
O componente principal 1 separou as amostras em relação ao aroma de chocolate, gosto ácido, aroma e sabor característico e aroma e gosto doce localizados no quadrante superior à direita (Figura 1a), enquanto que o aroma e sabor de cereal, corpo e adstringência variaram negativamente, podendo ser observados no lado esquerdo da referida Figura. $\mathrm{Na}$ segunda dimensão, as bebidas foram separadas de acordo com porcentagem aumento de grãos de café conilon, sendo reunidas nos seguintes grupos: bebida $\mathrm{A} 100$ e blends $\mathrm{C} 10$; $\mathrm{C} 20$ e C40; blend com C60 e C80 e bebida C100 (Figura 1b). O segundo componente foi dirigido pelos atributos de sabor e sensação na boca. Gosto amargo e numa menor extensão o aroma e sabor de velho tiveram cargas positivas enquanto a adstringência foi negativa nesta dimensão. A similaridade entre as bebidas A100 (100\% arábica) e C10 (arábica adicionada de $10 \%$ conilon) foi confirmada pela proximidade observada na Figura $1 \mathrm{~b}$ quadrante superior à direita.Entretanto, a bebida de grãos A100 foi 
caracterizada pelo atributo aroma de chocolate, e a bebida com grãos C10 pelos atributos aroma e sabor característico e aroma e gosto doce. Identificou-se semelhança entre as bebidas C40 e C60 localizadas no quadrante inferior à esquerda. A bebida $\mathrm{C} 100$ (100\% conilon) distinguiu-se sensorialmente das outras, ficando isolada no gráfico como pode ser observado no quadrante superior à esquerda.

Figura 1. Análise dos Componentes Principais (ACP) das bebidas de café, onde: (a) posição dos atributos. (b) posição das amostras e Mapa Interno da Preferência: (c) posição das amostras; (d) posição dos consumidores.

a)

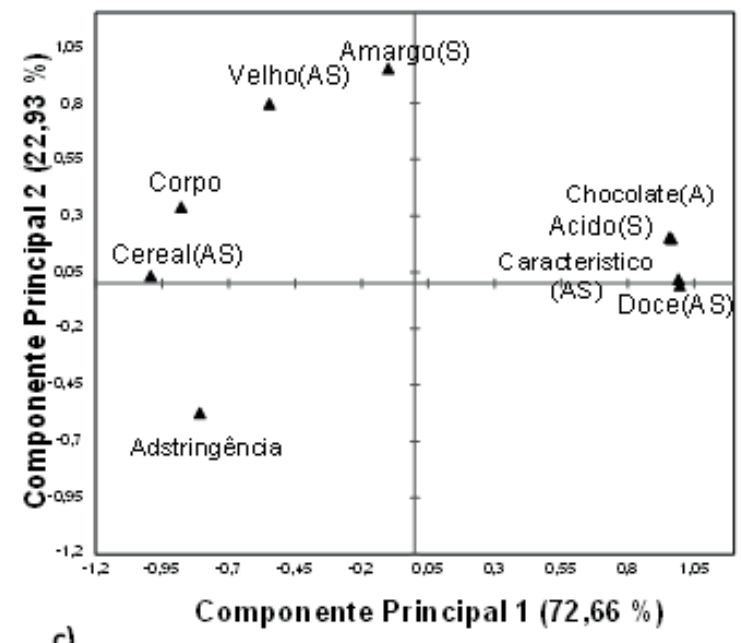

c)

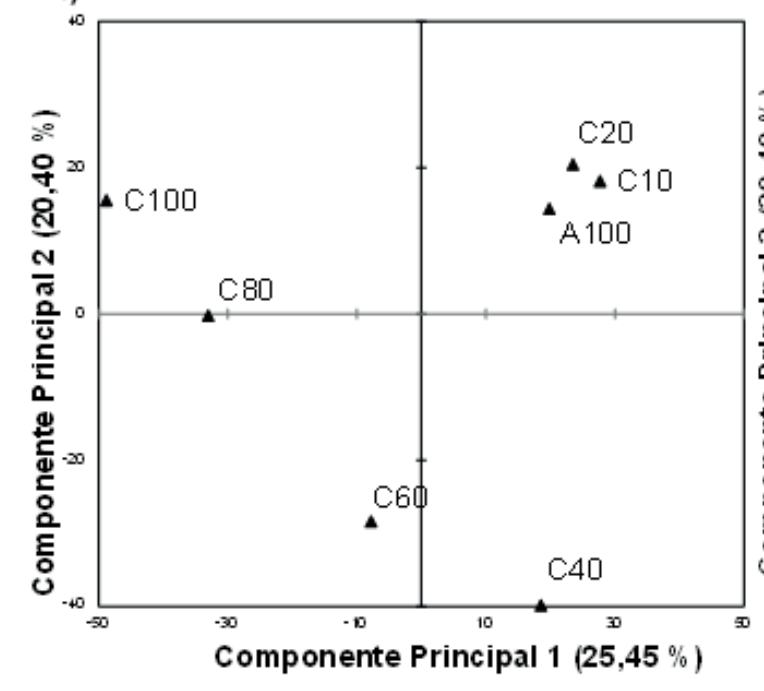

b)

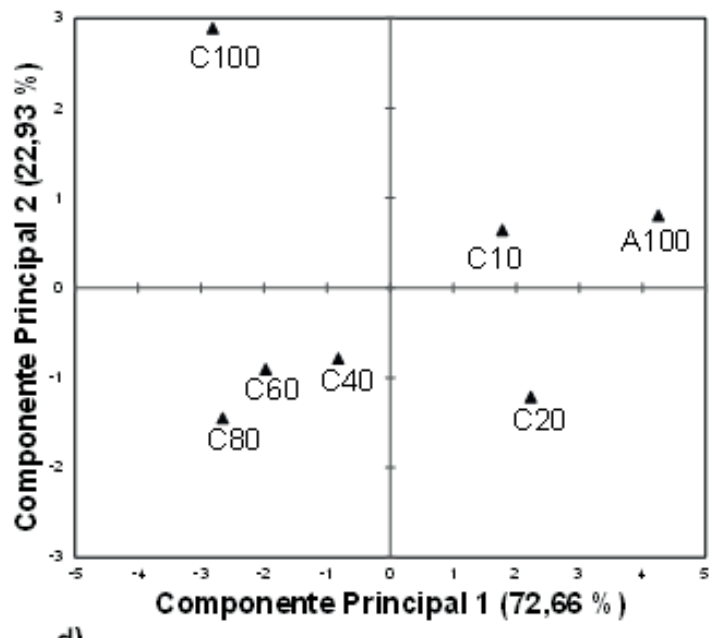

d)

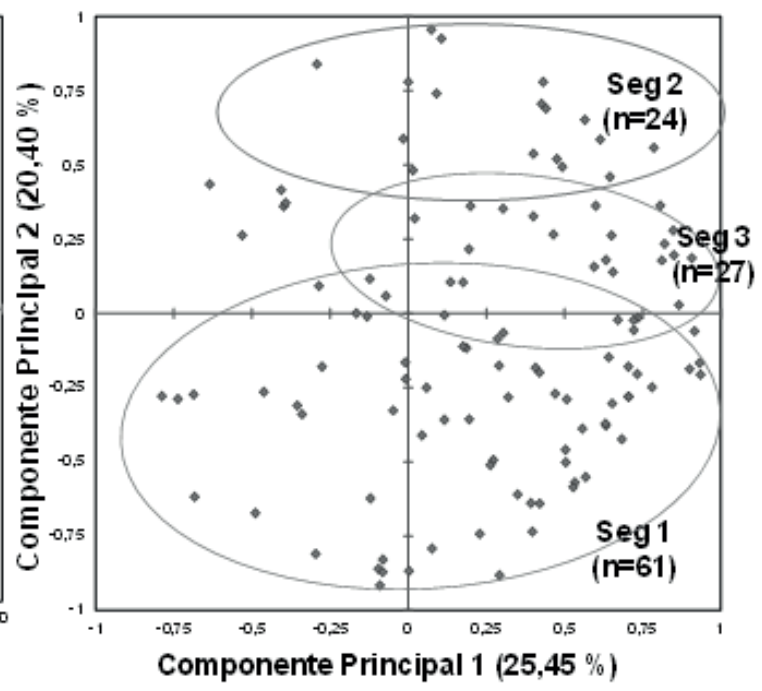

AS = Aroma e Sabor; $\mathrm{G}=$ Gosto; $\mathrm{A}=$ Aroma.

Fonte: Santos (2010).

As médias de aceitação dos consumidores alcançadas para as bebidas foram baixas e a adição de altas porcentagens de grãos conilon contribuiu negativamente para sua aceitação (Tabela 3). Entretanto, trata-se de resultados médios e devem ser analisados com cuidado. A preferência individual de cada consumidor é mostrada nas Figuras 1c e $1 \mathrm{~d}$. Os consumidores se alocaram nos quatro quadrantes da Figura 1d, confirmando a diversidade em relação à preferência das bebidas. A primeira dimensão (Figura 1c) separou as bebidas em relação ao conteúdo de grãos conilon, ou seja, aquelas com 
menores teores de conilon ficaram dispostas nos quadrantes da direita e com o aumento do conteúdo de grãos de café conilon as bebidas se alocaram à esquerda. $\mathrm{O}$ quadrante inferior e superior direito (Figura 1d) revelou a preferência para as bebidas C10, C20, C40 e A100.

Tabela 3. Aceitação ${ }^{\S}$ dos distintos segmentos de consumidores (média ${ }^{\S} \pm$ erro padrão) e média da aceitação pelos 112 consumidores (média ${ }^{\S} \pm$ erro padrão) para as bebidas de café preparadas com grãos conilon e arábica.

\begin{tabular}{lcccc}
\hline Amostras & $\begin{array}{c}\text { Segmento 1 } \\
(\mathbf{n}=\mathbf{6 1})\end{array}$ & $\begin{array}{c}\text { Segmento 2 } \\
(\mathbf{n}=\mathbf{2 4})\end{array}$ & $\begin{array}{c}\text { Segmento } 3 \\
(\mathbf{n}=\mathbf{2 7})\end{array}$ & Média (n=112) \\
\hline $100 \%$ arábica (A100) & $3,2^{\mathrm{cd}} \pm 0,26$ & $5,8^{\mathrm{ab}} \pm 0,47$ & $7,2^{\mathrm{a}} \pm 0,38$ & $5,4^{\mathrm{a}} \pm 0,26$ \\
$10 \%$ conilon (C10) & $3,4^{\mathrm{cd}} \pm 0,29$ & $7,0^{\mathrm{a}} \pm 0,46$ & $5,0^{\mathrm{b}} \pm 0,48$ & $5,1^{\mathrm{ab}} \pm 0,26$ \\
$20 \%$ conilon (C20) & $3,4^{\mathrm{cd}} \pm 0,30$ & $7,0^{\mathrm{a}} \pm 0,34$ & $6,5^{\mathrm{a}} \pm 0,35$ & $5,6^{\mathrm{a}} \pm 0,25$ \\
$40 \%$ conilon (C40) & $4,3^{\mathrm{ab}} \pm 0,32$ & $5,5^{\mathrm{b}} \pm 0,61$ & $5,2^{\mathrm{b}} \pm 0,51$ & $5,0^{\mathrm{ab}} \pm 0,25$ \\
$60 \%$ conilon (C60) & $4,5^{\mathrm{a}} \pm 0,32$ & $5,6^{\mathrm{b}} \pm 0,41$ & $4,0^{\mathrm{bc}} \pm 0,47$ & $4,7^{\mathrm{b}} \pm 0,23$ \\
$80 \%$ conilon $(\mathrm{C} 80)$ & $3,6^{\mathrm{bc}} \pm 0,31$ & $6,0^{\mathrm{ab}} \pm 0,41$ & $2,4^{\mathrm{c}} \pm 0,30$ & $4,0^{\mathrm{c}} \pm 0,24$ \\
$100 \%$ conilon $(\mathrm{C} 100)$ & $2,7^{\mathrm{d}} \pm 0,29$ & $5,8^{\mathrm{ab}} \pm 0,36$ & $2,9^{\mathrm{c}} \pm 0,35$ & $3,8^{\mathrm{c}} \pm 0,23$ \\
\hline
\end{tabular}

${ }^{\S}$ Avaliada em escala hedônica estruturada de 9 pontos, onde $1=$ desgostei extremamente; $9=$ gostei extremamente.

$\$$ Médias com letras iguais não diferem entre si $(p<0,05)$.

Fonte: Santos (2010).

Três grupos de consumidores foram identificados (Figura 1d), a saber: segmento 1 com 61, o segmento 2 com 24 e o segmento 3 com 27 consumidores, com distintas médias da aceitação (Tabela 3). Os consumidores do segmento 1 atribuíram maiores notas às bebidas C40 e C60 (4,3 e 4,5, respectivamente), embora, não tenham gostado de nenhuma das bebidas (médias abaixo de 5 na escala hedônica utilizada). Já o segmento 2 apreciou os blends $\mathrm{C} 10$ e C20, ambas com média 7,0 de aceitação e o segmento 3 preferiu as bebidas 100\% Arabica (A100:7,2) e C20: 6,5) com teores mais baixos de conilon. A baixa aceitação das bebidas de café, sobretudo pelos consumidores do segmento 1 , pode estar associada às características intrínsecas do produto, como o tipo de torrefação e o café utilizado para o preparo dos blends (os grãos de café conilon, por exemplo, foram Tipo 6), assim como características dos participantes, entre elas o consumo habitual de bebidas de maior ou menor qualidade em relação à das bebidas utilizadas no presente estudo. Mendes, Menezes e Da Silva (2001) mostraram que é possível otimizar a aceitação do café conilon em função da torrefação aplicada nos grãos, indicando a influência deste parâmetro na aceitabilidade do café por parte dos consumidores. Segundo Clarke e MacRae (1990) cada país possui um padrão de torrefação, sendo no Brasil, a comercialização do café torrado mais escuro.

A regressão dos dados sensoriais obtidos na ADQ com os dados hedônicos a partir da avaliação das bebidas pelos consumidores revela a preferência pelos segmentos bem como identifica os atributos sensoriais que dirigiram tal preferência (MAcFIE, 2007). Nas Figuras $2 \mathrm{a}$ e $2 \mathrm{~b}$ pode-se constatar que os consumidores do segmento 1 atribuíram notas mais elevadas às bebidas com porcentagem de grãos conilon intermediárias ( $\mathrm{C} 40$ e $\mathrm{C} 60$ ), as quais foram caracterizadas por maior adstringência e sabor de cereal. Os indivíduos dos segmentos 2 e 3 preferiram bebidas com menor quantidade de café conilon no blend, ou seja, preferindo aquelas com aroma de chocolate, aroma e sabor característico, aroma e gosto doce e gosto ácido. O segmento 3 foi o que mais rejeitou a adição de café conilon aos grãos arábica, tendo sido observado significativo decréscimo nas 
médias da preferência a partir de C20. Tal resultado sugere que os consumidores deste segmento provavelmente estão mais habituados às bebidas com teor maior de café arábica e reconheceram a presença de café conilon nos blends. Mendes (2005) constatou que a bebida usada em seu estudo equivalente à C40 foi a segunda bebida eleita pelos consumidores em relação ao ideal de sabor e a bebida com $100 \%$ grãos arábica (A100) um sabor muito abaixo do ideal. A superfície do contorno (Figura 2c) gerada a partir do Mapa da Preferência apresenta quantos segmentos têm a preferência acima da média para determinada região do Mapa. As bebidas A100, C10 e C20 foram preferidas pelos segmentos 2 e 3 , os quais se situam numa região de 60 a $80 \%$ de probabilidade de aceitação acima da média da preferência. A partir do perfil sensorial e da avaliação da aceitação do consumidor, identificou-se atributos importantes que dirigiram a preferência, revelando resultados que podem ser empregados no setor cafeeiro, a fim de disponibilizar no mercado, dentro de uma nova estrutura competitiva, blends que sejam apreciados e satisfaçam as necessidades de um maior número de indivíduos para a bebida de café.

Figura 2. Mapa Externo da Preferência das bebidas de café mostrando: (a) posição das amostras e dos três segmentos de consumidores, (b) posição dos atributos sensoriais definidos na ADQ e (c) superfície de contorno das bebidas de café por segmentos de consumidores. AS = Aroma e Sabor/Gosto; A = Aroma.

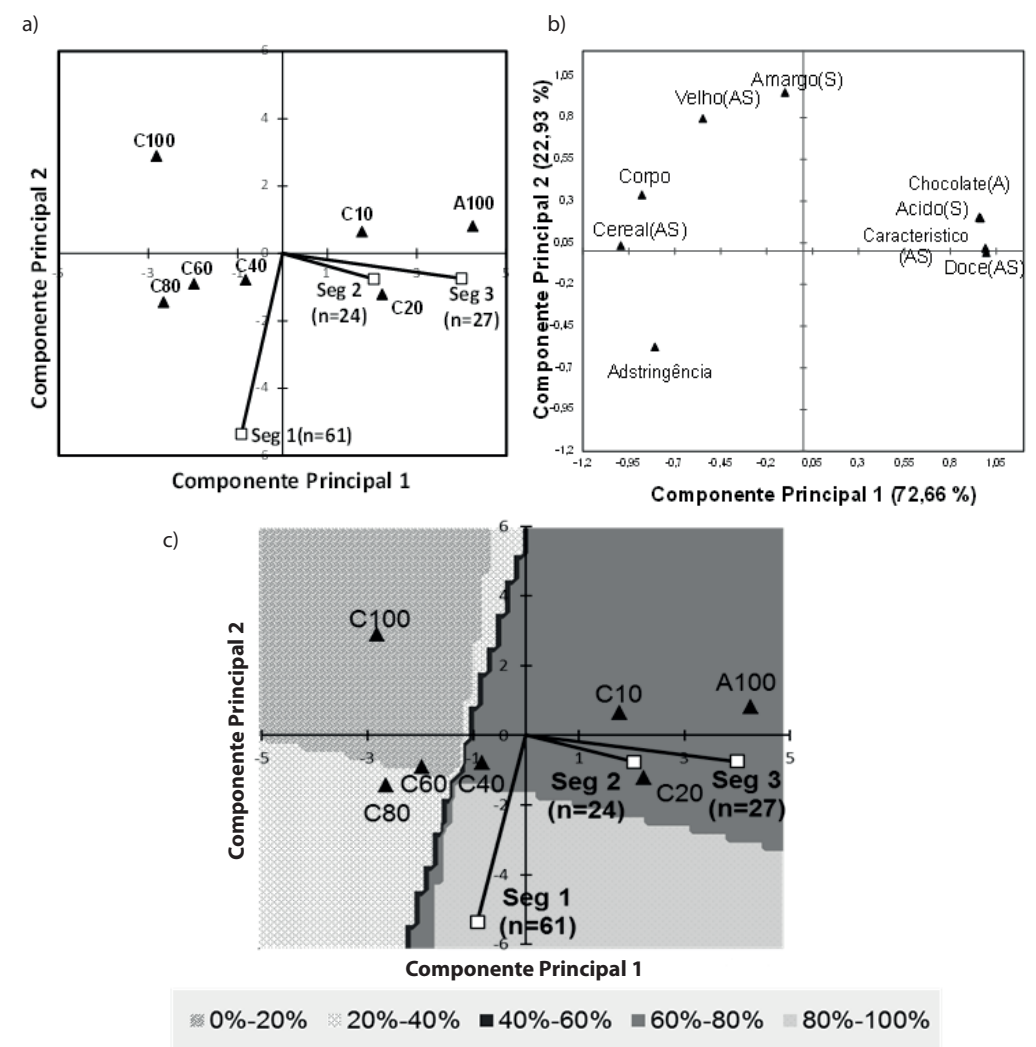

Fonte: Santos (2010).

\section{Conclusões}

A ADQ demonstrou que o aumento da quantidade de grãos conilon nos blends intensificou o aroma e sabor de cereal (atributo presente em maior intensidade no café conilon). Além disso, houve uma tendência de redução na intensidade do 
aroma de chocolate, aroma e sabor característico, aroma e gosto doce e ácido. De acordo com as condições experimentais do presente estudo, as bebidas com até $20 \%$ de grãos conilon mantiveram características sensoriais tradicionalmente positivas na bebida, como atributos mais intensos de aroma chocolate, aroma e sabor característico de café, aroma e gosto doce.

\section{Agradecimentos}

Os autores agradecem ao CBP\&D Café pelo apoio financeiro e ao $\mathrm{CNPq}$ pela bolsa de estudos ao primeiro autor.

\section{Referências}

ADDINSOFT, S. A. R. L. XLSTAT software, version 9.0. Paris, France, 2009.

ARRUDA, A. C.; FERREIRA, M. A. M.; MINIM, V. P. R. Perfil dos consumidores de café de Viçosa/MG: Um estudo exploratório. In: SIMPÓSIO DE PESQUISAS DE CAFÉS DO BRASIL, 5., 2007, Águas de Lindóia. Anais... Águas de Lindóia: [s.n], 2007. CD-ROM.

BHUMIRATANA, N.; ADHIKARI, K.; CAHAMBERS IV, E. Evolution of sensory aroma atributes from coffee beans to brewed coffee. LWT - Food Science and Technology, London, v. 44, n. 1, p. 2185-2192, 2011.

BIOSYSTEM. FIZZ Sensory analysis and consumer test management software. Version 2.10, Biosystemes, Couternon, France, 2005.

CHALFOUN, S. M.; PEREIRA, M. C.; CARVALHO, G. R.; PEREIRA, A. A.; SAVIAN, T. V.; BOTELHO, D. M. S. Sensorial characteristics of coffee (Coffea arábica L.) varieties in the alto Paranaíba region. Coffee Science, Lavras, v. 8, n. 1, p. 43-52, 2013.

CLARKE, R. J.; MACRAE, R. Coffee chemistry. Londres: Elsevier Applied Science, v. 1, 1990. 320 p.

CLIFFORD, M. D. Chemical and physical aspects of green coffee and coffee products. In: CLIFFORD, M. D.; WILLSON, K. Coffee: botany, biochemistry and production of beans and beverage. London \& Sydney: CROOM HELM, 1985. cap. 13.

COCHRAN, W. G.; COX, G. M. Experimental designs. 2. ed. London, John Wiley, 1957. 611 p.

DAMÁSIO, M. H.; COSTELL, E. Analisis sensorial descriptivo: Generación de descriptors y selección de catadores. Revista de Agroquímica y Tecnología de Alimentos, Madri, v. 31, n. 2, p. 165-178, 1991.

DE MARIA, C. A. B.; TRUGO, L. C.; MOREIRA, R. F. A. PETRACCO, M. Simultaneous determination of total chlorogenic acids, trigonelline and caffeine in green coffee samples by high performance gel filtration chromatography. Food Chemistry, London, v. 52, n. 4, p. 447-449, 1995.

GREENHOFF, K.; MACFIE, H. J. H. Preference mapping in practice. In: MacFIE, H.; THOMSON, D. M. H. (Ed.). Measurement of food preferences. London: Blackie Academic \& Professional, 1994. cap. 6, p. 137166.

HOUGH, G.; WAKELING, I.; MUCCI, A.; CHAMBERS IV, E.; GALLARDO, I. M.; ALVES, L. R. Number of consumers necessary for sensory acceptability tests. Food Quality and Preference, Barking, v. 17, n. 6, p. 522526, 2006.

MacFIE, H. J. H. Assessment of the sensory properties of food. Nutrition Reviews, New York, v. 48, n. 2, p. 87-93, 1990.

MacFIE, H. J.; BRATCHELL, N.; GREENHOFF, K.; VALLIS, L. V. Designs to balance the effect of order of presentation and first-order carry-over effects in hall tests. Journal of Sensory Studies, Connecticut, v. 4, n. 2, p. 129-148, 1989.

MacFIE, H. J. Preference mapping and food product development. In: Macfie, H. (Ed.). Consumer-led food product development. Cambridge: CRC Press, Woodhead Publishing Limited, 2007. p. 551-592.

MEILGAARD, M.; CIVILlE, C. V.; CARR, B. T. Sensory evaluation techniques. Florida: CRC Press, Inc., 1999.

MENDES, L. C. Estudos para determinação das melhores formulações de blend de café arábica (Coffea arabica) com café robusta (Coffea canephora Conillon) para uso no setor de cafés torrados e moídos espresso. 2005. Tese (Doutorado em Tecnologia de Alimentos) Universidade Estadual de Campinas, Campinas.

MENDES, L. C.; MENEZES, H. C.; DA SILVA, M. A. A. P. Optimization of the roasting of robusta cofee $(C$. canephora conillon) using acceptability tests and RSM. Food Quality and Preference, Barking, v. 12, n. 1, p. 153162, 2001.

MOURA, S. C. S. R.; GERMER, S. P. M.; ANJOS, V. D. A.; MORI, E. E. M.; MATTOSO, L. H. C.; FIRMINO, A.; NASCIMENTO, C. J. F. Avaliações físicas, químicas e sensoriais de blend de café arábica com café canephora 
(Robusta). Brazilian Journal of Food Technology, Campinas, v. 10, n. 4, p. 271-277, out./dez. 2007.

PANGBORN, R. M. Influence of water composition, extraction procedures, and holding time and temperature on quality of coffee beverage. LWT - Food Science and Technology, Amsterdam, v. 15, n. 3, p. 161-168, 1982.

PEREIRA, R. C. A.; SOUZA, J. M. L.; AZEVEDO, K. S.; SALES, F. Obtenção de café com qualidade no Acre. Rio Branco: Embrapa Acre, 2000. 27 p. (Circular técnica, 34).

SANTOS, E. S. M. Perfil sensorial e aceitabilidade do consumidor para blends de bebida de café preparadas com grãos arábica (Coffea arábica L.) e conilon (Coffea canéfora P.). 2010. Dissertação (Mestrado em Ciência e Tecnologia de Alimentos) - Universidade Federal Rural do Rio de Janeiro, Seropédica.

SANZ, C.; MAEZTU, L.; ZAPELENA, M. J.; BELLO, J. Profiles of volatile compounds and sensory analysis of three blends of coffee: influence of different proportions of arabica and Robusta and influence of roasting coffee with sugar. Journal of the Science of Food and Agriculture, Davis, v. 82, n. 8, p. 840-847, 2002.

SCHLICH, P.; McEWAN, J. A. Preference mapping: a statistical tool for the food industry. Science des Aliments, Cachan, v. 12, n. 3, p. 339-355, 1992.
SCHOLZ, M. B. S.; SILVA, J. V. N.; FIGUEIREDO, V. R. G.; KITZBERGER, C. S. G. Atributos sensoriais e características físico-químicas de bebida de cultivares de café do IAPAR. Coffee Science, Lavras, v. 8, n. 1, p. 6-16, 2013.

STAUB, C. Agtron/SCAA roast classification. Color disk system. Chicago: Specialty Coffee Association of, 1995.

STONE, H.; SIDEL, J. L. Sensory evaluation practices. Londres: Academic Press, 2004. 311 p.

STONE, H. S.; SIDEL, J. L.; WOOSLEY, A.; SINGLETON, R. C. Sensory evaluation by quantitative descriptive analysis. Food Technology, Chicago, v. 28, n. 11, p. 24-34, 1974

$\mathrm{R}$ Development Core Team. R: A language and environment for statistical computing. R Foundation for Statistical Computing, Vienna, Austria. 2009. Disponível em: $<$ http://www.R-project.org $>$. Acesso em: 26 jun. 2013.

WESTAD, F.; HERSLETH, M.; LEA, P. Strategies for consumer segmentation with applications on preference data. Food Quality and Preference, Barking, v. 15, n. 7-8, p. 681-687, 2004. 